\title{
Registries in Accountable Care
}

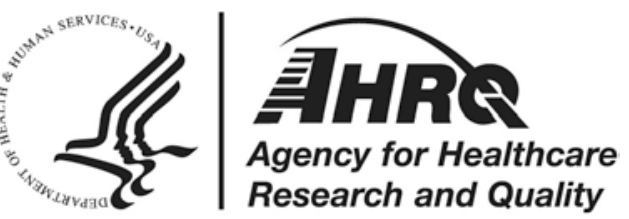




\section{Research White Paper}

\section{Registries in Accountable Care}

\section{Addendum to Registries for Evaluating Patient Outcomes: A User's Guide, Third Edition}

Prepared for:

Agency for Healthcare Research and Quality

U.S. Department of Health and Human Services

5600 Fishers Lane

Rockville, MD 20857

www.ahrq.gov

Contract No. 290-2014-00004-C

Prepared by:

L\&M Policy Research, LLC

Washington, DC

Author:

John Sharp, MSSA, PMP, FHIMSS

AHRQ Publication No. 17(18)-EHC026-EF

February 2018

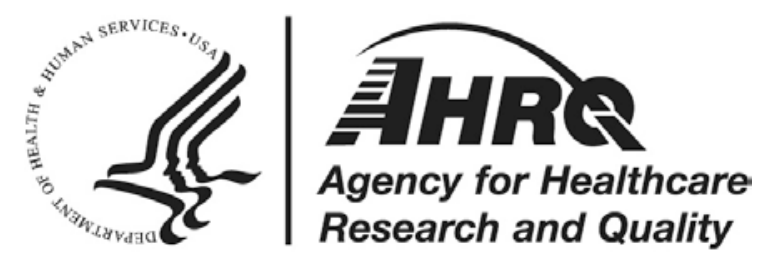


The Effective Health Care Program of the Agency for Healthcare Research and Quality (AHRQ) conducts and supports research focused on the outcomes, effectiveness, comparative clinical effectiveness, and appropriateness of pharmaceuticals, devices, and health care services. More information on the Effective Health Care Program can be found at www.effectivehealthcare.ahrq.gov.

This report is based on research conducted by L\&M Policy Research, LLC, with partners OM1 and IQVIA, under contract to the Agency for Healthcare Research and Quality, Rockville, MD (Contract No. 290-2014-00004-C). The findings and conclusions in this document are those of the authors, who are responsible for its contents; the findings and conclusions do not necessarily represent the views of AHRQ. Therefore, no statement in this report should be construed as an official position of AHRQ or of the U.S. Department of Health and Human Services.

\section{None of the authors have any affiliations or financial involvement that conflicts with the material presented in this report.}

This report may be used and reprinted without permission except those copyrighted materials that are clearly noted in the report. Further reproduction of those copyrighted materials is prohibited without the express permission of copyright holders.

Persons using assistive technology may not be able to fully access information in this report. For assistance contact EPC@ahrq.hhs.gov.

Suggested citation: Sharp, J. Registries in Accountable Care. White Paper, addendum to Registries for Evaluating Patient Outcomes: A User’s Guide, Third Edition. (Prepared by L\&M Policy Research, LLC, under Contract No. 290-2014-00004-C.) AHRQ Publication No. 17(18)EHC026-EF. Rockville, MD: Agency for Healthcare Research and Quality; February

2018. www.effectivehealthcare.ahrq.gov. DOI: https://doi.org/10.23970/AHRQREGISTRIESACCOUNTABLE. 


\section{Reviewers (alphabetical):}

James Colbert, M.D.

Harvard Medical School

ACO Learning Network at The Brookings Institution

Michelle B. Leavy, M.P.H.

OM1

Nancy A. Dreyer, Ph.D., M.P.H., FISPE

Chief of Scientific Affairs \& Head

Center for Advanced Evidence Generation, IQVIA

Richard E. Gliklich, M.D.

Chief Executive Officer and Chairman, OM1

Steven J. Spalding, M.D.

Cleveland Clinic 


\section{Contents}

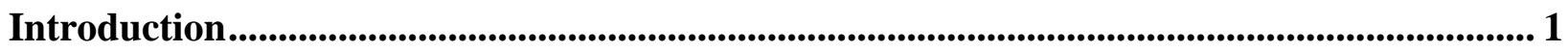

Role of Registries in Accountable Care .................................................................................................... 2

Types of Accountable Care Arrangements ................................................................................... 2

Registries Versus Other Approaches …………………....................................................... 3

Goals of Registries in Accountable Care ................................................................................. 5

Population and Care Management ........................................................................................ 5

Registry Use in Monitoring Quality Metrics................................................................ 7

Controlling Costs and Improving Efficiency ...................................................................... 7

Designing Accountable Care Registries ....................................................................................................... 8

Registry Purpose and Objectives .......................................................................................... 8

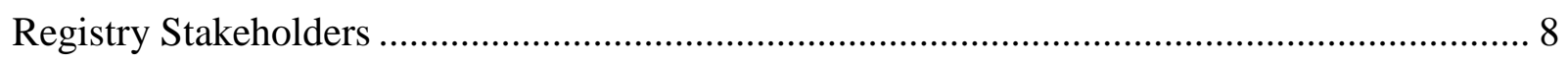

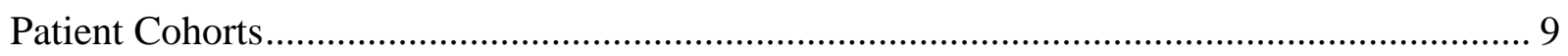

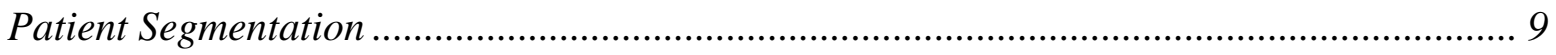

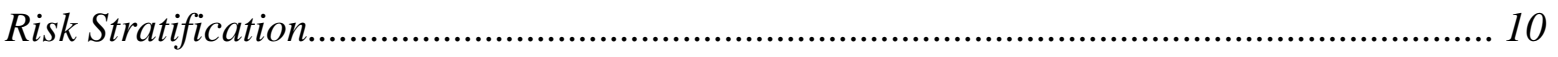

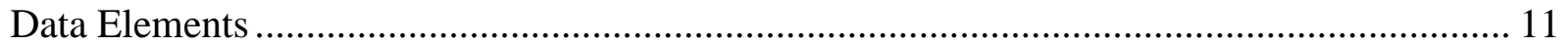

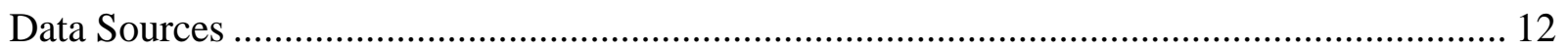

Data Architecture and Models …………………………..................................................... 13

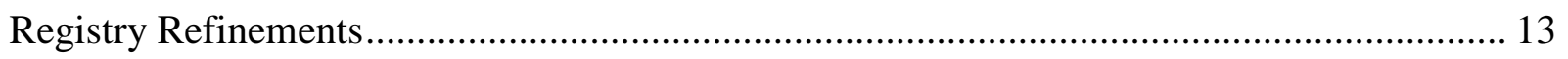

Transforming Registry Data Into Knowledge...................................................................................... 14

Dashboards and Data Visualization ...................................................................................... 15

Self-Service Analytics ................................................................................................... 15

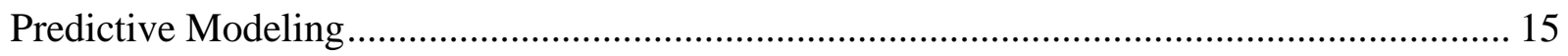

Future Role for Use of Registries in Accountable Care ................................................................... 16

Changing Structure and Data Needs ...................................................................................... 16

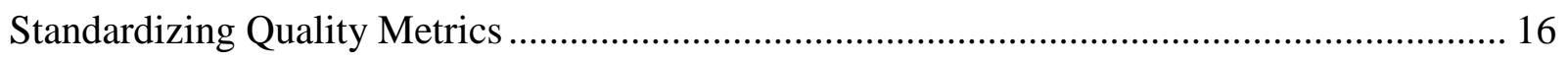

Best Practices and Innovation in Registries....................................................................... 17

Policy and Research Implications....................................................................................... 17

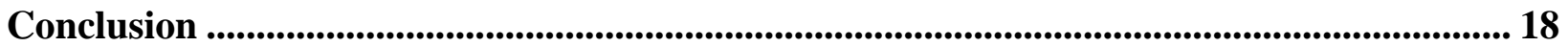


Registries in Accountable Care

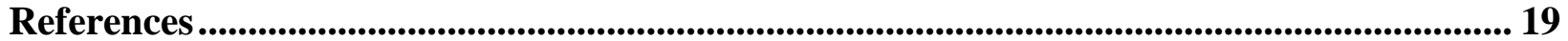

Table

Table 1. Types of accountable care arrangements ............................................................. 2 
AUTHOR'S NOTE: The description of the Centers for Medicare \& Medicaid Services' (CMS) portfolio of accountable care organization (ACO) models was current at the time this paper was written in 2015. In the intervening years, the existing ACO models have evolved and new models have been introduced. A current list of CMS payment and service delivery models can be accessed at: https://innovation.cms.gov/initiatives/\#views=models. Similarly, examples of specific health care organizations presented in this paper and the accompanying descriptions, which include reference to size, network configuration, operating model, and other characteristics, were current at the time the author wrote the paper. Any one or more of these elements may have changed over time.

\section{Introduction}

Patient registries, when properly designed and conducted, can provide unique insights into real world clinical practice and quality of care, assist in care delivery and coordination, and support quality improvement. A patient registry is defined as “....an organized system that uses observational study methods to collect uniform data (clinical and other) to evaluate specified outcomes for a population defined by a particular disease, condition, or exposure, and that serves one or more predetermined scientific, clinical, or policy purposes." ${ }^{11}$ While many registries are designed to fulfill research purposes, registries have also proven useful for tracking patients with specific conditions, identifying gaps in care, and measuring both short- and long-term patient outcomes.

With their ability to focus on specific populations and adapt to changing requirements, registries can be powerful tools to support accountable care organizations (ACOs), as well as other organizations participating in accountable care arrangements. The Centers for Medicare \& Medicaid Services (CMS) defines ACOs as "health care providers, who come together voluntarily to give coordinated high quality care to their Medicare patients... [ensuring] that patients, especially the chronically ill, get the right care at the right time, while avoiding unnecessary duplication of services and preventing medical errors." ${ }^{2}$ Many types of organizations, including hospital systems, physician groups, and integrated delivery systems, are working to deliver accountable care by integrating new payment models. These payment models generally focus on three separate aims originally described by the Institute for Health Improvement: (1) improving the patient experience of care; (2) improving the health of populations; and (3) reducing the per capita cost of health care. ${ }^{3}$ At least two experts predicted that by 2020, a majority of reimbursement for hospitals will be through ACOs and other risksharing arrangements. ${ }^{4}$

To meet these aims, ACOs and other organizations participating in accountable care arrangements must be able to combine clinical and financial data with analytic capabilities. Registries provide an approach to integrate data from multiple sources (both data collected for other purposes and data collected primarily for the registry) and facilitate the use of these data to segment the patient population into appropriate cohorts, conduct risk stratification, and generate the types of actionable information necessary to achieve the goals of accountable care. Registries that track patient outcomes - in particular long-term and/or patient-reported outcomes - and registries that provide benchmarking capabilities on regional, national, or international levels have great potential for helping ACOs and other organizations improve performance. 
This paper provides a brief overview of accountable care arrangements and then describes the role of registries in supporting the goals of accountable care, considerations for developing and operating an accountable care registry, and trends that may affect the use of these types of registries in the near future.

\section{Role of Registries in Accountable Care}

\section{Types of Accountable Care Arrangements}

Accountable care arrangements represent a variety of different partnerships between health care organizations, payers, and providers designed to improve care coordination and individual patient experience, improve health outcomes, and reduce health care expenses. Table 1 describes the widely recognized accountable care arrangements currently in existence, although variations in these arrangements and models continue to be developed as organizations and payers strive to transition to value-based care.

\section{Table 1. Types of accountable care arrangements}

\begin{tabular}{|c|c|}
\hline $\begin{array}{l}\text { Type of Accountable } \\
\text { Care Arrangement }\end{array}$ & Description \\
\hline $\begin{array}{l}\text { Medicare Pioneer } \\
\text { Accountable Care } \\
\text { Organizations (ACOs) }\end{array}$ & $\begin{array}{l}\text { An initiative of the Centers for Medicare \& Medicaid Services (CMS) Center for Medicare } \\
\& \text { Medicaid Innovation (CMMI) designed to test an alternative payment method. A limited } \\
\text { number of care delivery systems were selected by CMMI to participate in the Pioneer } \\
\text { ACO Program based on demonstrated experience providing patient-centric, integrated } \\
\text { care. The demonstration began on January } 1,2012 \text {, and during the first } 2 \text { years } \\
\text { organizations that met quality goals and achieved a specified level of cost savings } \\
\text { received a shared savings payment. Starting in year three of the program, some } \\
\text { organizations elected to move towards population-based payments and capitation. } \\
\text { Participating organizations also were required to develop risk-sharing arrangements with } \\
\text { commercial insurance payers such that greater than } 50 \text { percent of the organization's } \\
\text { revenue will eventually be derived from alternative payment methods. }\end{array}$ \\
\hline \multirow{4}{*}{$\begin{array}{l}\text { Medicare Shared } \\
\text { Savings Program } \\
\text { (MSSP) ACOs }\end{array}$} & $\begin{array}{l}\text { A permanent Medicare program mandated by the Affordable Care Act offering a pathway } \\
\text { for groups of health care providers to become ACOs. }\end{array}$ \\
\hline & $\begin{array}{l}\text { To achieve shared savings, participating organizations must submit data on } 33 \text { quality } \\
\text { measures and reduce overall spending by more than } 2 \% \text { from a pre-defined benchmark. }\end{array}$ \\
\hline & $\begin{array}{l}\text { MSSP ACOs can choose between one-sided and two-sided risk arrangements. In the } \\
\text { one-sided risk arrangement, an ACO can receive shared savings but is not responsible } \\
\text { for paying Medicare if total costs exceed the benchmark. In the two-sided risk } \\
\text { arrangement, the ACO has the potential to receive a higher amount of shared savings, } \\
\text { but the ACO must pay Medicare if costs are higher than a set benchmark. }\end{array}$ \\
\hline & $\begin{array}{l}\text { The Advance Payment (AP) ACO Model provides a select group of MSSP-eligible } \\
\text { providers start-up funding to support necessary infrastructure and care coordination } \\
\text { work. The AP ACO Model is tailored for physician-based and rural providers. }{ }^{a}\end{array}$ \\
\hline $\begin{array}{l}\text { Commercial ACO } \\
\text { Arrangements }\end{array}$ & $\begin{array}{l}\text { Many shared savings arrangements exist between provider organizations and } \\
\text { commercial insurance companies. Each commercial insurer has its own policies and } \\
\text { rules for quality measures and cost savings that must be met in order to receive shared } \\
\text { savings. }\end{array}$ \\
\hline $\begin{array}{l}\text { Managed Care } \\
\text { Arrangements }\end{array}$ & $\begin{array}{l}\text { Managed care organizations receive a per-member-per-month fee to assume } \\
\text { responsibility for total cost of care for patients. Managed care arrangements exist within } \\
\text { Medicare, Medicaid, and commercial insurance companies. }\end{array}$ \\
\hline
\end{tabular}




\begin{tabular}{|c|c|}
\hline $\begin{array}{c}\text { Type of Accountable } \\
\text { Care Arrangement }\end{array}$ & Description \\
\hline $\begin{array}{l}\text { Bundled Payment } \\
\text { Arrangements }\end{array}$ & $\begin{array}{l}\text { Bundled payments entail reimbursement to health care providers and larger } \\
\text { organizations, including providers, for expected costs of specific episodes of care over } \\
\text { time. Bundled payments represent an intermediary payment model between fee-for- } \\
\text { service and capitation (i.e., full risk arrangements). }\end{array}$ \\
\hline
\end{tabular}

Multiple types of organizations participate in accountable care arrangements. For example, the Medicare Pioneer ACOs are typically large, integrated systems, several of which participated in prior CMS demonstration programs. In comparison, organizations participating in the Medicare Shared Savings Program range from integrated delivery systems that include hospitals, primary care providers, and specialists to physician-led organizations that only include primary care providers who work in the outpatient setting. Commercial ACO, managed care, and bundled payment arrangements also include multiple types of organizations.

\section{Registries Versus Other Approaches}

A central goal of accountable care arrangements is to improve the overall health of the population covered within a given arrangement. To achieve this goal, organizations typically leverage multiple sources of clinical and financial data to identify gaps in care, track improvement efforts, and monitor the covered population. Three common approaches to using these data to improve performance are electronic health record (EHR) systems, data warehouses, and patient registries.

EHR systems are transactional systems designed to document patient care. These systems contain detailed clinical data about patient care and may be useful for understanding treatment patterns, identifying gaps in care, and providing decision support tools at the point of care. However, EHRs do not contain all of the information necessary to support efforts to improve patient experience and population health. For example, EHRs often do not contain data on patient-reported outcomes or information on issues important to patients, such as ability to return to work.

Paper-based systems present a greater challenge. Data must be manually abstracted into an electronic system or data transfer application using an agreed upon format which can be subsequently imported into the registry and/or EHR. This can be an expensive process, although many practices that continue to depend on paper medical charts have billing software with claims data that can provide a portion of the needed data elements to facilitate care coordination.

Many organizations that are participating in accountable care arrangements are using more than one EHR system; for example, a hospital may use an inpatient EHR system, while the physician groups affiliated with the hospital use different ambulatory EHR systems. The use of multiple EHR systems, sometimes in combination with paper-based systems, makes it difficult to track populations with specific conditions or gaps in care across one organization, and even more so when multiple organizations are involved. 
A logical solution for multiple EHR systems is to utilize a health information exchange (HIE) if a functional one exists. This assumes that the HIE was established with the agreement to share data for analysis and not just for treatment or coordination of care. In the absence of a fully functional HIE, an entity must negotiate data sharing agreements and ensure patients have given permission to transfer their data to another institution. Data formats must be agreed upon using a common standard for exchanging health care information electronically such as ConsolidatedClinical Document Architecture (C-CDA) or, the Fast Healthcare Interoperability Resources (FHIR) specification, an alternative standard drafted in 2012 in support of electronic health information exchange.

A data warehouse is another option for supporting the goals of accountable care. It comprises an end-to-end solution for extracting data from primary sources to providing query and reporting capabilities. Many health care organizations are adopting data warehouse and business intelligence technologies in the quest to become data-driven providers. However, a system-wide data warehouse likely emphasizes the ability to produce broad operational or financial reports for the organization as a whole with potentially different data requirements than those of accountable care entities. While a data warehouse is typically designed to help analyze and report on data from disparate sources, it may be insufficient for addressing all of the data needs of an accountable care initiative.

In contrast to EHR systems and data warehouses, dedicated registries allow the accountable care initiative to focus on its own data requirements for quality and operational metrics and provide a platform for data mining of the population of interest. Registries offer a vital means of tracking key characteristics of these populations that need to be monitored in order for the organization and its care teams to ensure an entire population (sometimes without regard to health status) is getting the care needed to keep them as healthy as possible. Even prior to the rise in accountable care arrangements, some organizations used disease- and condition-specific registries for clinical care and management of specific patients. As the organizations have evolved their focus towards population health under accountable care arrangements, these existing registries are sometimes incorporated into a larger suite of accountable care tools. Registries are being used to both target interventions for population and care management, as well as to support quality measurement and monitoring and to help control or reduce the costs of doing so. In addition, registries are particularly useful in organizations with multiple EHR systems, where the registry can overlay the EHR systems as a single repository for both the subset of data extracted from the EHRs and other systems and the primary data collected for the purpose of the registry.

HIEs can provide an opportunity to obtain information on ACO patients who receive care from providers, hospitals and emergency departments (EDs) outside of the ACO provider network. This requires both syntactic interoperability (the ability to exchange data) and semantic interoperability (the ability to understand the exchanged data). ${ }^{5}$ At least two conditions must be present to use HIEs successfully in an ACO registry. The first condition is that the HIE agreement between provider organizations must include not only use of the data for treatment but also use of the data for the registry. Second, the data need to feed seamlessly into the registry. This means that the registry should accept data in the C-CDA format (or in the future, FHIR specification). Alternatively, the data must be transferred to the receiving provider's EHR and then extracted to the registry with other EHR data on that patient. An ongoing challenge for HIEs is patient matching which entails ensuring that the patient for whom the data is requested 
matches the patient in the registry. Given the absence of a national patient matching strategy, individual provider and institution-level matching strategies will continue to be the norm. ${ }^{6}$

\section{Goals of Registries in Accountable Care}

Within the context of accountable care, registries are typically defined by a population that has been attributed to a particular provider organization for the purposes of accountability for cost and quality, whether for a given episode of care or all care required to keep a defined population healthy. These registries are used to identify gaps in care, such as screening services that have not been provided but would be appropriate for a given population, or monitoring of a clinical condition that should occur and where a patient has yet to come in for care. These registries sometimes include financial information on patients, specifically; payer claims data, unlike research or public health registries. Accountable care registries often focus on a specific population within a cohort that receives most of their care from one health system so that interventions and measures can be consistently applied by participating providers. The following sections discuss specific goals of registries in accountable care.

\section{Population and Care Management}

Accountable care arrangements address health management for whole populations, including those who are healthy, those with chronic diseases, and those at high risk for complications. The literature suggests that registries are a key element of an information technology strategy for population health management. In a 2010 paper from the Agency for Healthcare Research and Quality (AHRQ), the authors state that "having access to an EHR or registry increases the likelihood that practices are performing the functionalities" of population health management. Specifically, the authors note that widespread adoption of population health management requires "technological innovations; greater availability of usable data."7 A recent report identified several steps in population health management that could be informed by the information generated by a registry, including: (1) identifying subpopulations of interest; (2) examining detailed characteristics of identified subpopulations; (3) creating reminders for patients and providers; and (4) tracking performance measures. ${ }^{8}$ The first three of these steps are described below, while tracking performance measures is discussed in the following section.

Because of the wide range of patients included in accountable care arrangements, the ability to identify and actively manage subpopulations is of critical importance. Organizations participating in accountable care arrangements, in their pursuit of efficient and improved care delivery and population health management, can use registry data to identify specific patients in a given population who would benefit from additional clinical services or care coordination services. For example, Bon Secours Medical Group, a multispecialty group practice of over 600 providers that is part of a multi-state health system that includes hospitals and long-term care facilities, is an ACO participating in the Medicare Shared Savings Program (MSSP), as well as in accountable care arrangements with commercial payers. The group uses multiple registries to manage patient care, with a particular focus on patient care after hospital discharge or ED visits to prevent avoidable readmissions and inappropriate ED use. Patients are segmented into registries by diagnoses, such as chronic heart failure, acute myocardial infarction, and pneumonia, and the group maintains a high-risk registry for complex patients. Through use of the registries as well as other initiatives, the group has reduced readmission rates to 2.08 percent in 
the past two years. ${ }^{9}$ Bon Secours' five long-term care facilities contributed to the lowering of readmissions, reducing their hospital readmission rate by 22 percent over the prior year. ${ }^{10,11}$

Examining subpopulations such as those with multiple conditions and expensive treatments is another key function of patient registries. Crystal Run Healthcare, an independent multi-specialty group practice with over 300 providers based in New York, uses a registry to support care coordination efforts for complex patients, such as those who are repeatedly readmitted to the hospital or those who are frequent utilizers of emergency department services. Because the entire practice uses a single EHR system, the registry can take data directly from the EHR and combine it with claims data for reporting and care management purposes. The registry offers the ability for the organization to analyze data at the provider level, as well as examine each provider's patient panel to identify patients for care coordination or followup. Follow-up efforts include care coordinators making home visits for patients recently discharged from acute care settings, as well as arranging for follow-up appointments with patients with complex care needs. The organization has achieved a lower cost of care, which was within the Medicare minimum savings rate, and decreased inpatient utilization in the first 21 months of participation in the MSSP. ${ }^{12}$

Registries may be particularly useful for management of patients with complex care needs. In a paper from The Commonwealth Fund, the authors define the concept of complex care management as, "programs in which specially trained, multidisciplinary teams coordinate closely with primary care teams to meet the needs of patients with multiple chronic conditions or advanced illness, many of whom face social or economic barriers in accessing services.” The report implies the need for a registry that not only includes data on previous health care utilization by high cost patients but also data from comprehensive health assessments and behavioral and social factors to support care management for these types of patients. ${ }^{13}$

The creation of reminders for providers and patients is another aspect of population health management, and more specifically, care management, that can be facilitated through the use of patient registries. Clinical guidelines can be used as the basis for automated reminder systems that prompt providers and patients when a visit, exam, or procedure is due. Several studies have documented the effectiveness of reminder systems for reducing missed visits, improving medication adherence, and increasing rates of immunization. For example, a recent study found that automated text message appointment reminders improved the likelihood of patients attending scheduled outpatient follow-up visits after an ED discharge. ${ }^{14}$ A 2014 study found that automated telephone reminders improved adherence with once-daily glaucoma medications, ${ }^{15}$ while a systematic review found that reminder systems increase adherence for patients taking asthma medications. ${ }^{16}$ Reminders are also useful for increasing immunization rates; one study found that automated text message reminders were associated with increased influenza vaccination among pregnant women, ${ }^{17}$ while findings from one study support the use of text message reminders to improve human papillomavirus vaccine series completion rates. ${ }^{18}$ As is evident in these examples, reminders may be sent to patients in multiple modes (e.g., email, text, telephone, mail), the choice of which may depend on patient preferences and/or available contact information. 


\section{Registry Use in Monitoring Quality Metrics}

Accountable care arrangements and payer contracts typically include specific performance measures, including measurements of quality of care. For example, CMS established 33 quality metrics $^{\mathrm{a} 1}$ to help gauge Pioneer and MSSP ACO performance and outcomes. These measures span four domains: Patient/Caregiver Experience, Care Coordination/Patient Safety, Preventive Health, and At-Risk Populations, the last of which consists of five measures and two composites: diabetes, hypertension, ischemic vascular disease, heart failure and coronary artery disease. ${ }^{19}$ The diabetes measure is a single measure plus a composite of five measures and the coronary artery disease metric is a composite consisting of two measures.

Commercial payers also have uniquely-defined metrics, again including quality of care, focusing on their specific populations of interest. For example, commercial ACO arrangements may address pediatric quality metrics, whereas Medicare does not cover this population. Even measures that address the same clinical area (e.g., 30-day readmission for heart failure) may be defined differently for different payers. These discrepancies in measures and their definitions place additional financial and logistical burdens on health care delivery organizations to maintain informatics, analytics systems and multiple registries to meet reporting requirements. There is a clear need for harmonization of measures, as discussed in the 'Future Role of Registries within Accountable Care' section below.

To enable monitoring these quality metrics, registries can be used to collect key clinical variables that describe the numerator and denominator of given quality metrics as defined by CMS or other payers. Reporting capabilities within the registry may allow the organization to track progress on the quality metrics in between reporting periods.

\section{Controlling Costs and Improving Efficiency}

Considerations surrounding efficiency include minimizing instances in which patients over- and under-utilize health care. Accountable care arrangements are intended to provide for better coordination between providers and across settings, which can facilitate reductions in redundant and unnecessary care and improve the efficiency of care delivery. In addition, the registries, if updated at least daily, can provide a view into the patient's current status and care plan, thereby helping both primary care and specialist providers coordinate the patient's care and develop a more comprehensive care plan.

Registry use in accountable care can offer a view of the population for a group of providers to focus resources on patients who need them the most to improve their health status. For instance, prioritizing patients for invasive procedures can be based on disease status rather than scheduling availability. In a Health Affairs blog, the authors state that ACOs assume that ED utilization and inpatient stays will drop when compared to non-ACO members. They also suggest that, an ACO may implement efficiencies such as same day scheduling for all patients; so for these high

\footnotetext{
${ }^{a}$ CMS published a final rule to the Shared Savings Program for ACOs on June 9, 2015. The rule changed several program areas including beneficiary assignment, data sharing, available performance risk models, eligibility requirements, participation agreement renewals, and compliance and monitoring. See https://www.cms.gov/
} 
performing ACOs, it is better to manage the MSSP with their commercial shared savings contracts to reap benefits for all patients under these arrangements. ${ }^{20}$

Tests and procedures for specific conditions do not need to be repeated when the data on each patient are available to the provider through EHR systems and registries, thereby increasing system efficiencies and eliminating redundancies. In addition, through registries, providers can be benchmarked for their utilization of tests and procedures as a means to educate on and encourage more cost efficient behaviors. Improving efficiency and quality can have a substantial impact on costs, as demonstrated by Intermountain Healthcare in Utah, a system with 22 hospitals and a medical group with more than 185 physician clinics. Intermountain leveraged electronic health data to measure, analyze, and provide feedback to clinicians about clinical variations and outcomes. Using process metrics, the system was able to reduce costs and improve clinical outcomes across a number of areas, including obstetrics and acute respiratory distress. ${ }^{21}$

\section{Designing Accountable Care Registries}

Designing an accountable care registry to meet the goals described in the previous section will require following the steps outlined in the section on "Creating Registries" in the AHRQ registries guide. ${ }^{1}$ This section describes best practices for planning and designing a registry, as well as selecting data elements, incorporating other data sources, and collecting patient-reported outcome measures. When moving through these steps, designers of accountable care registries should consider several factors unique to these types of registries.

\section{Registry Purpose and Objectives}

Developing a new patient registry for an ACO can be daunting. One of the first steps is to clearly state the purpose and objectives of the registry. Accountable care registries may fulfill multiple purposes for an organization. To be effective and cost-efficient, these registries must fit within the workflow of the organization, meaning that they provide actionable information and do not duplicate the output of other systems. Registry developers need to design these registries anticipating change, from a governance, funding and information technology perspective, as accountable care arrangements evolve. Further, it is critical to assess which function(s) the registry will play within the health system, in comparison to other existing systems. Clearly stating the purpose and objectives of the registry, as well as the value proposition for the registry at the outset will enable the registry developers to focus on collecting the most relevant data and generating useful reports. For instance, care coordination staff often need working lists of patients who are likely to be at high risk without follow-up. Examples may include daily reports on patients discharged from a hospital within the past week with diagnoses at high risk for readmission or lists of patients within the practice who are diagnosed with type 2 diabetes but have not had a recent HbA1c or eye exam. These lists can be used to identify patients that require follow up by care coordinators or a primary care team member.

\section{Registry Stakeholders}

As part of defining the purpose and objectives of an accountable care registry, it is important to identify and involve the stakeholders of the proposed registry. The stakeholder group may be large and diverse, particularly if the registry is intended to meet multiple needs within the organization. Identifying and incorporating feedback from stakeholders throughout the process 
will help ensure that the resulting registry is feasible and meets the needs of these stakeholders. Ongoing collaboration can also help encourage provider use of and support for the registry.

The Cleveland Clinic Health System, a large multi-specialty academic medical center with over 3,000 physicians and scientists, recently used a stakeholder-driven process to design and build a registry for monitoring the initial 33 measures required by the CMS ACO initiatives. To develop the registry, Cleveland Clinic organized a large stakeholder group, including representatives from information technology, quality, primary care, finance, and analytics that worked together over an 18-month period to develop definitions for the measures and attribution models. The stakeholder group also developed data governance and change management standards and procedures. As noted by the registry developers, the success of the technical development of the registry was completely dependent on early collaboration with clinical providers and strong leadership from the administrative and clinical leadership teams. ${ }^{22}$

\section{Patient Cohorts}

Accountable care arrangements typically focus on subsets, or cohorts, of patients, such as patients with specific diseases or risk factors, which must be clearly defined during the registry design phase. Cohorts of patients can be defined from within the larger patient population based on specific population health objectives and interventions (such as specific disease management programs or care management of patients with multiple chronic conditions). Often, a patient cohort is defined by the presence of a chronic disease such as diabetes or heart disease. A cohort can also be defined by procedures, including orthopedic procedures for risk-based commercial contracts that pay for short-term outcomes or episodes of care including surgery, rehabilitation and homecare. High utilizer cohorts may be defined as a subset within these larger cohorts, such as patients with frequent admissions or ED use or other high cost care. ${ }^{13}$

\section{Patient Segmentation}

Patient segmentation refers to the process of using population health registries to select specific groups of patients with common characteristics. The goal of segmenting patients by cohort is to then provide such patients specific interventions if needed (including, but not limited to, home monitoring, diabetes education, and access to care managers and health coaches). The ability to execute queries on patient registries and identify particular patient cohorts is essential for organizations seeking to provide high quality care to an entire population. Examples include identifying all patients with chronic heart failure who have had two or more hospital admissions in the past 6 months or patients with diabetes with an HbA1c of greater than nine who are not taking insulin. Once identified, those patients can be enrolled in a home-based heart failure management program or scheduled for visits with a diabetes pharmacist or educator. The more specific the patient cohort, the more targeted of an intervention the organization can deploy.

The first step in population health interventions is often the identification of a specific patient cohort for whom a given intervention would help improve their health, quality of care, patient experience and potentially control per capita health expenditures. In accountable care arrangements, areas of focus for an organization are frequently influenced by payer performance metrics associated with managing conditions such as diabetes, heart disease, and lung disease, especially chronic obstructive pulmonary disease. However, a cohort could also encompass 
disease categories by focusing on high utilization or risk scores. For instance, the Sutter Care Coordination Program defines a complex patient by any one of these factors: unplanned readmission within 30 days, two or more admissions in the past year, two or more ED visits in the past year, seven or more medications, or three or more chronic conditions. ${ }^{13}$ Some cohorts may be determined by a specific risk score based on these types of factors or more advanced stages of a chronic condition, such as, uncontrolled hypertension or Stage 2 or 3 chronic kidney disease. In identifying a cohort based on a chronic condition, it is not prudent to use a single laboratory value or test result as the sole determining factor. For instance, to determine advanced chronic kidney disease, two glomerular filtration rate (GFR) results confirming the diagnosis are recommended. ${ }^{23}$

While chronic disease state is the most common criteria for cohort definition, demographics can also play a role. Age can be a factor, particularly for Medicare ACOs. Geographic characteristics such as limiting a cohort to a specific city or region, urban versus rural, or even census track can be important for some organizations that are limiting their population by location of their patients or estimated socio-economic status which is closely linked to social determinants of health. Patient segmentation can also occur by insurance type as larger health systems may be working under multiple accountable care arrangements and contracts.

\section{Risk Stratification}

Risk stratification of patients is key to success in managing a population. Risk stratification differs from patient segmentation in that patient segmentation may include defining groups by variables other than risk including demographics and treatment types. The data required to generate these risk estimates is highly dependent on how risk is defined. Risk can refer to generic outcome measures such as overall payer spend (e.g., per member per month) or mortality. Risk may also refer to more specific outcomes such as risk of 30- or 90-day readmission, ED utilization, or medication noncompliance. It is important that organizations working towards accountable care determine what risk they are attempting to manage in their initial phase of operation prior to building a registry. Risk can be defined initially by using historical data from the defined patient population and categorizing patients by risk level. For instance, one report posits three levels of utilization risk: the low risk patient, the rising-risk patient and the high-risk patient. ${ }^{24}$ Rising risk refers to patients who have multiple risk factors that could elevate them into the high cost category if unaddressed. Some organizations choose to focus on the rising-risk patients anticipating that they will have a greater ability to change this group's behavior and outcomes.

Numerous proprietary algorithms to identify high risk and high cost patients exist. ${ }^{25}$ One example of a non-proprietary predictive model is the Johns Hopkins Adjusted Clinical Risk Groups. This model uses a five-step process to develop Resource Utilization Bands and Reference Concurrent Weights for the elderly and non-elderly and decision trees. ${ }^{26}$

Publicly available analytic algorithms also exist. The CMS Hierarchical Condition Categories (HCCs), developed with Medicare and Medicaid data, are used to create predictive ratios and categorize beneficiaries into deciles and percentiles of predicted annualized expenditures. The HCC model includes comparison tables showing predictive ratios for body system or disease group categories, such as end stage renal disease. The Charlson Comorbidity Index is another 
publicly available algorithm that takes into account major chronic conditions and age to create a score or probability. It has been well validated and used widely in studies of chronic disease. ${ }^{27}$

These predictive models and data points enable the organizations focusing on accountable care to evaluate risk and manage it more effectively.

\section{Data Elements}

An early step in registry development is identifying a basic set of data elements, consistent with the purpose and objectives of the patient registry. The numerators and denominators of quality metrics identified by the accountable care team or its payers often will drive the data elements to be collected in a registry designed to support accountable care goals.

The National Quality Measures Clearinghouse (NQMC) has a Domain Framework that can assist in selecting data elements for a registry to ensure the availability of numerators and denominators for quality measures. The organization divides measures related to health into delivery measures and population health measures. For delivery, they include clinical quality measures, related health care delivery measures and clinical efficiency measures. For population health, NQMC includes population health quality measures, related population health measures and population health efficiency measures. For discrete data elements, such as laboratory results, data can be collected longitudinally but also selected to be included in measures by specific time intervals. Clinical observations may be recorded as the presence or absence or degree of a symptom and these observations need to be recorded at the point of care as discrete data elements (i.e., field-defined) rather than within clinical notes or narrative.

In addition to quality measures, efficiency measures are often important for accountable care organizations. Data elements for efficiency measures typically include health care utilization, such as, admissions, readmissions, and ED visits. Other utilization and delivery of care data elements can include frequency of tests and procedures, transitions in care, homecare services and prescription activity. ${ }^{28}$ Payer spend data, reported as paid claims, can also be used as a broader measure of efficiency and can be useful to include as registry elements and categories.

While the basic data set for registries may focus on data elements for specific measures, data that trigger alerts (reminders) for providers as part of clinical decision support (CDS) can also be included in registries. Organizations managing accountable care arrangements can utilize data to identify workflow and daily alerts on high risk patients or procedures by generating daily reports on the patient population. "Increased sophistication around data element 'attributes' is needed so CDS systems can assign, alert and suggest actions for responsible entities to take.” Care planning can also determine data and interoperability standards; this could include patient diagnosis, orders (interventions, services, procedures) and care goals (patient and provider-specific outcomes, and actual outcomes). ${ }^{29}$

Organizations focusing on accountable care, whether they are ACOs or other entities trying to implement bundled payments, have acknowledged the importance of monitoring patient engagement and patient-reported data in reaching their quality and cost goals. ${ }^{30}$ Measurement of patient engagement and patient-reported outcomes can provide valuable information to enable more effective identification of patients in need of additional support and ensure that population 
health registries remain patient-centric. The Patient Activation Measure is the most widely studied and validated instrument for measurement of patient engagement with medical care, and research has shown that patients with higher activation scores have lower health care costs than those who are less engaged in their care. ${ }^{31}$ Other data sometimes available within the category of patient engagement include data from remote monitoring devices, patient accessing a patient portal (including their own data), patient health status questionnaires, and online patient education. $^{30}$

Examples of patient-reported data include surveying patients regarding their pain and mobility following knee-replacement surgery or monitoring patients with depression via a validated instrument such as the Patient Health Questionnaire-9. Integrating the results of these surveys into population health registries can allow clinicians and care managers to better track outcomes that are important to patients. Operationally, such surveys can be conducted in person, in a clinic waiting room, over the telephone, via mail, or electronically through use of a secure website, when conducted with patients who are not cognitively impaired. Patient-reported outcomes (PROs) are a subset of patient-reported data that may be collected as part of a registry. Validated PROs are available for many condition areas and should be selected based on the intended use of the information, taking into consideration the potential burden for the patient. ${ }^{1}$

Patient experience measures are also an important aspect of accountable care. Most accountable care payer arrangements (including Medicare) include Clinician and Group or Hospital Consumer Assessment of Healthcare Providers and Systems (CAHPS) survey results. These can be reported for the population as a whole or for patients covered by the accountable care arrangement only. Integrating these external data presents a challenge since these measures are collected by entities external to the organization and may intentionally be de-identified to protect patients' privacy.

Registries may also collect information on other factors that affect both outcomes and costs. For example, registries may collect information on interventions, such as interventional radiology procedures, surgeries, and administration of intravenous medications including chemotherapy and physical or occupational therapy. Interventions may also include health coaching, offers of social and financial supports, scheduling follow up visits to ensure certain laboratory results are regularly conducted, medication reconciliation or telephonic or home visits within one week following discharge from an inpatient setting. Information on specific care plans, such as, physical therapy for a certain number of weeks or a schedule of followup appointments, may also be included in the registries.

\section{Data Sources}

One of the challenges in accountable care arrangements is collecting, integrating and normalizing data from other clinical entities that are treating the patient. In some cases, all of the care may be confined to one health system and recorded in a single EHR system so long as patients utilize only those providers or EDs affiliated with the system or accountable care entity. Many patients are also seen in specialty hospitals, urgent care centers, post-acute facilities and pharmacies not affiliated with an accountable care entity or system that uses a common EHR, resulting in a multitude of data sources. Key data primary care data elements can also be found in non-participating pharmacies and retail health clinics (examples include vaccinations and on-site 
immediate, non-urgent care) or at opticians (examples include eye exams for patients with diabetes) as well as primary care physician offices. While some facilities with EHRs are becoming part of HIEs so that data can be acquired for patients within a given population, this is not yet the norm, and incorporating key data points from multiple sources into registries is a challenge.

Accountable care registries may also incorporate claims data received from payers such as Medicare and commercial payers. For instance, Bon Secours Medical Group currently operates a separate registry with Medicare claims data for their ACO patients and is evaluating new analytics platforms to combine the data and enable analysis of clinical data from their EHR system in conjunction with claims data. However, these data may not be available until several months after the date of service, making them less useful for both assessing and addressing current risks within the patient population.

\section{Data Architecture and Models}

Increasingly, data warehousing and business intelligence tools are being adopted by health care organizations. While registries to support accountable care may be separate from an organization-wide data warehouse, registry development can benefit from the expertise within the organization on data acquisition, data transformation, and data storage. Teams that support disease-specific registries that are multi-institutional may also maintain registries for accountable care purposes. Typically, registries for accountable care rely on a standard star (or star-join) schema in a relational database. This database would extract data from multiple sources including payers, practice management systems, clinical information systems and health risk assessments. Subsequently, data would be the normalized and integrated from these multiple sources and then validated. The registry would have a mechanism to add newly eligible patients to the cohort and remove those no longer eligible (for example, patients lost to follow up). ${ }^{30}$ The database would require a security scheme conforming to Health Insurance Portability and Accountability Act (HIPAA) regulations so that access to identifiable data is authorized only for providers and staff members with appropriate credentials, for the purposes of clinical decision support. Data governance and information management principles and tools should be implemented to ensure these data are of high quality and fidelity.

In some cases, databases other than a relational database would be indicated. For instance, some business analytics companies are utilizing Not Only Structured Query Language (NoSQL) databases using technology solutions such as, Hadoop, to manage large amounts of data. ${ }^{32}$ Other organizations are using technology called Late Binding to enable flexibility in data mapping in development of their architectural models. ${ }^{33}$ These architectures offer the ability to house extremely large sets of data in a loosely or unstructured fashion and then leverage coding and queries to build on-the-fly data sets for analysis. The effectiveness of these approaches to data management for accountable care registries will be borne out over time.

\section{Registry Refinements}

Changes to the registry are required on a periodic basis, especially as individual ACO contracts transition to new contract periods. First, the governance group, typically made up of physicians, practice managers, payer contract managers, business intelligence experts, and information 
technology experts, would evaluate the contract changes and the implications for data elements, registry structure, and reports. Funding for the registry must also be evaluated periodically to assess return on investment and projecting when a breakeven point might be reached or if personnel changes are required. Because frequent changes in the registry may be necessary, a flexible architecture for the registry is required with a change management process that can respond to these changes. Close integration with the business intelligence/data warehouse team can enable the registry team to be aware of changes in the EHR and other data sources. As part of responding to frequent changes, a communication and training process should be in place to communicate with end users (providers and care managers). The registry must be built in a manner that makes it flexible to accommodate frequent changes both from a governance and technology standpoint.

\section{Transforming Registry Data Into Knowledge}

A variety of tools can be employed to transform registry data into knowledge using analytics and CDS for the purposes of managing a patient population. First, basic reports can be generated which provide summary statistics on the patient population stratified by both health and economic (cost) risk factors. Reports can be scheduled to run at pre-determined times or run on demand. Another type of basic report is a patient list report that is populated based on combination of patient characteristics. This could include a list of patients currently part of a given cohort by provider. Lists of patients who no longer qualify for the cohort or are new to the registry could be identified to inform the providers about this change in status. Examples of other reports might include a list of potentially high risk patients and summary statistics on those who have not had a follow up appointment in the past six months or those discharged from acute care within the past 30 days. These list-based reports can then be used for the care team to follow up with patients to address active medical issues.

Quality measures and outcome measures can also be the focus of reports generated using registries. For instance, reports may provide information on how many patients are currently in a numerator or denominator between reporting periods, to gauge how close an organization is to reaching specific quality targets. Cost reports showing expenditures by cohort (e.g., setting, disease, comorbidities, procedures, etc. as recorded in registries) could also be generated to evaluate how an organization is meeting financial targets or where to focus future interventions to improve care, population health, or control expenditures.

Utilizing data from a registry to analyze costs can provide different perspectives on the ability of an accountable care entity to monitor areas of health care expenditures and manage organizational finances. Also, cost avoidance analysis can evaluate whether there is a reduction in unnecessary procedures, duplicate tests, ED visits or few hospitalizations. Opportunity costs are another type of cost report. Efficacy of programmatic interventions can also be derived from these data; for instance, if an organization initiates two chronic care programs aimed at reducing acute care utilization for patients, data contained within the accountable care registry can compare clinical outcome and utilization data to guide the comparison. 


\section{Dashboards and Data Visualization}

Current business intelligence tools such as dashboards represent an important role in patient management within accountable care arrangements as well as in other contexts. Dashboards are best utilized to examine retrospective or real-time data, some of which can be provided via registries. Besides summary statistics, guided-drill down capabilities are particularly important in being able to visualize data at the practice group level, individual provider level and patient level. This way, providers can compare their performance with others in the group and see their individual performance. In addition, the detailed reports and registries behind dashboards can be used to identify individual patients who are not meeting quality benchmarks. Care coordinators and other support staff may also find dashboard technology valuable. As care plans are usually executed using a team approach, other disciplines like nurse practitioners, physician assistants, and case managers will require an individualized visualization strategy that aligns with their role on the care team.

The timely availability of data also requires real-time or near-real-time reporting. Data must be available as soon as possible to provide for ongoing care planning and identifying new risks that may appear. The challenges of providing real-time data to a registry are considerable. ExtractTransfer-Load (ETL) processes must be designed to not only transfer data from the EHR and other systems but also ensure the reliability and timeliness of the data for continuous use.

As an example, the Cleveland Clinic registry described above provides a data mart that allows providers to see quality measure results. Measure results are available at the enterprise, facility, division, department, and individual provider level. Providers also have access to a central portal where they can examine their own compliance with measures, as well as drill down to detailed lists to determine which patients are out of compliance with specific quality measures. Administrative and clinical leadership can access a dashboard to review more aggregate level reporting across the organization. Self-service analytic tools, which are discussed in the next section, are also available. While it is too early to quantify outcomes associated with registry use, utilization of the data mart and its reporting and visualization tools has been good to date, with many providers regularly using the portal to review their results and identify and close gaps in care. $^{22}$

\section{Self-Service Analytics}

Another helpful tool in accountable care arrangements is self-service analytics. Beyond standardized reports and dashboards, the goal of self-service analytics is similar to that of clinical research informatics. Tools that allow self-guided discovery of data within the registry can enable the organizations focusing on accountable care delivery to find novel relationships between data that may influence the design of patient segmentation and risk models and possibly identify factors that affect outcomes.

\section{Predictive Modeling}

Moving beyond self-service analytics is the development of predictive models. Utilizing the registry data, new algorithms can be analyzed and tested to identify new risk groups and potential care paths that might improve outcomes. Access to large data sets from the registry can be analyzed by data scientists, either within the organization or through contracted vendors, who 
run models, such as Monte Carlo simulations, multiple times to find meaningful predictive models and develop algorithms to be used in CDS. One type of CDS using these algorithms is a clinical risk calculator. Many organizations may not have the ability to create their own predictive models but will utilize existing tools, such as the Framingham Coronary Heart Disease Risk Score and the World Health Organization Fracture Risk Assessment Tool Score as well as those described in the risk stratification section above. These risk calculators, algorithms, and models serve to reduce important decision-influencing clinical care to key data points to identify risk utilizing the registry data. They also can be incorporated into the EHR and care processes. By identifying risk and quality issues within the point of care through EHR technology, care efficiency can be improved and outcomes impacted.

\section{Future Role for Use of Registries in Accountable Care}

\section{Changing Structure and Data Needs}

Registry architecture, functions, and capabilities will evolve as ACOs and other accountable care payment arrangements mature. Since these arrangements are in the early stages of growth and payment models are just beginning to shift, registries supporting them must remain flexible as new types of patient segmentation and algorithms emerge. For instance, MSSPs initially focused on a limited set measures and conditions, but this set almost certainly will be refined and expanded over time to include other chronic disease conditions. In addition to organizations tracking more chronic conditions through registries over time, some organizations are recognizing the value of using other patient segmentation methods; geographic location, in particular, is emerging as an area of interest following a report on especially high cost patients, or "super utilizers.",34

\section{Standardizing Quality Metrics}

One major challenge for registries in accountable care is the variation in quality measure definitions. Medicare ACOs largely use the National Quality Forum (NQF) as their basis for quality measures with standardized numerators and denominators well defined. Concurrently, commercial payers may have different quality metrics that do not conform to the NQF clearinghouse of quality measures, but rather are tailored to address the individual payers' areas of concern. These variations place a substantial financial and logistical burden on provider organizations, and both government payers and commercial payers need to come to consensus on what should be measured to ensure accountable care. This consensus would simplify the management of registries by requiring a more limited set of common data elements.

Standardizing metrics could also support care coordination. When a team working with a specific population has consensus on the targeted quality metrics, all can be working toward the same goals rather than disparate purposes.

Two entities working on quality metric harmonization are the National Quality Forum's Measure Applications Partnership and CheckQM, a private measure harmonization effort. In addition, the National Committee for Quality Assurance (NCQA) now has an accreditation program to measure ACO performance. Specifically, NCQA requests reports on core performance and patient experience measures to determine if ACOs are "integrating and using data to provide high quality, well-coordinated, patient-centered care and identify where [they] can improve quality and efficiency". ${ }^{29}$ These measures include Healthcare Effectiveness Data and Information 
Set (HEDIS) measures and metrics for all age groups, including children and efficiency measures and patient surveys. NCQA also addresses the issue of patient attribution; that is, showing results of only patients covered, either those covered by a specific contract or those who receive 50 percent or more of their care from the provider organization. While these efforts are in their early stages, they are intended to help to simplify the number of and incongruities between measures including those from Medicare and commercial accountable care arrangements.

\section{Best Practices and Innovation in Registries}

Successful models and best practices for use of registries in accountable care will emerge as ACOs and other forms of value-based health care delivery evolve. Along with best practices in care paths, best practices in the use of registries must also be documented and be modified to address unique factors associated with given populations, systems, and cultures. Data management is key to the success of identifying patients at risk, supporting care models and report quality outcomes for any organization focusing on delivering accountable care. These early successful registries within these organizations, such as Medicare Pioneer ACOs, will become the basis for best practices in data management and use.

Beyond best practices, this early phase of development for registries in accountable care will promote innovation in data management as well as care models and outcome measurement. The data from registries needs to be available at the point of care through creative visualizations, on mobile devices and integrated into standardized care algorithms and EHRs. Several EHR and analytics companies are developing solutions for each of these innovations. Data visualization in healthcare is advancing rapidly and will become one of the major innovations in displaying registry data in ways that provide optimal CDS.

Learning communities are beginning to develop around best practices for ACOs. Examples include the Patient Centered Primary Care Collaborative (https://www.pcpcc.org/) and the ACO Learning Network (http://www.acolearningnetwork.org/), which has a workgroup on Effectively Integrating and Using Health IT. These communities can help new or struggling ACOs learn best practices from those with established and successful registries.

\section{Policy and Research Implications}

There are significant policy implications for registry development such as House Resolution 5214 (H.R.5214): "To require the Secretary of Health and Human Services to provide for recommendations for the development and use of clinical data registries for the improvement of patient care.” Policy can also drive the standardization of quality measures noted above. The recent report authored by JASON, an independent group of scientists that advises the Federal government on matters of science and technology, for the Office of the National Coordinator for Health IT, provides additional support for data standardization in interoperability which could enable organizations using accountable care registries to acquire and standardize data more routinely. A recent JASON report indicated that to provide a path toward realizing the promise of a robust health data infrastructure, a unifying health information technology architecture must be developed that adheres to core principles, such as, "use public APIs [application programming interfaces] and open standards, interfaces, and protocols, include with the data the corresponding 
metadata, context, and provenance information, and represent the data as atomic data with associated metadata., 35

There are also research implications for use of accountable care registries. Data mining of clinical data is a growing enterprise, particularly in academic medical centers. This data mining can lead to new predictive models and algorithms to understand difficult challenges, such as how to prevent readmissions in congestive heart failure patients or which patients are most at risk for an ED visit and what factors might prevent high cost utilization. Clinical research informatics can also contribute to better measurement of outcomes including those that are patient-reported. Research can facilitate intervention design and make recommendations on how to improve interventions or how new interventions, such as telemedicine, influence the outcomes of patients and healthy populations in accountable care arrangements. ACO registries can also be used for rapid cycle patient-oriented outcomes research, efforts that are supported by the Patient-Centered Outcomes Research Institute.

While the use of these data for research purposes offers much potential, it should be noted that research activities might introduce the need for ethical review. Accountable care registries generally fit under the Health Insurance Portability and Accountability Act definition of health care operations. When research studies are introduced, questions may arise as to whether the study falls under the Common Rule (federal policy on the protection of human subjects), and whether informed consent is required. This issue has been explored at length with regard to quality improvement registries. Further details are provided in the chapter on "Quality Improvement Registries” in the AHRQ registries guide. ${ }^{1}$

\section{Conclusion}

Registries are a vital element in the management and success of organizations seeking to shift toward value-based health care delivery by being accountable not only for controlling medical costs but also for improving quality and population health. Without careful data management, organizations will lack the tools to report on high risk patients and quality outcomes. The proper implementation of a registry that includes the appropriate and validated data elements provides a platform for development of reports, dashboards, and visualizations to impact clinical decision support.

Since organizations striving to be accountable for population health are in their infancy, a centralized repository of registry models, including those that are proprietary, would help to advance the shift toward value-based health care delivery. In addition, movement toward consensus on the necessary quality metrics to support accountable care, and development of standardized data elements as a foundation for these quality metrics, are critical steps to support the aggregation of registry data and national benchmarking, which is essential for understanding value. The sharing of algorithms and predictive models through academic publications and opensource software promotion will also aid in the achievement of the three aims of accountable care payment models - improving patient experiences and population health and while at the same time controlling per capita health care costs. 


\section{References}

1. Gliklich R, Dreyer N, Leavy M, eds. Registries for Evaluating Patient Outcomes: A User's Guide. Third edition. Two volumes. (Prepared by the Outcome DEcIDE Center [Outcome Sciences, Inc., a Quintiles company] under Contract No. 290 200500351 TO7.) AHRQ Publication No. 13(14)-EHC111. Rockville, MD: Agency for Healthcare Research and Quality. April 2014. http://www.effectivehealthcare.ahrq.g ov/registries-guide-3.cfm.

2. Centers for Medicare \& Medicaid Services. Accountable Care Organizations (ACOs). http://www.cms.gov/Medicare/MedicareFee-for-Service-Payment/ACO/. Accessed August 2, 2015.

3. Institute for Healthcare Improvement. IHI Triple Aim Initiative. http://www.ihi.org/Engage/Initiatives/Triple Aim/pages/default.aspx. Accessed August 2. 2015

4. Emanuel EJ and Liebman JB. The end of health insurance companies [Blog post]. January 30, 2012. http://nyti.ms/1ez5idK. Accessed August 2, 2015.

5. "Case Example 34. Technical and security issues in creating a health information exchange." In: Gliklich R, Dreyer N, Leavy M, eds. Registries for Evaluating Patient Outcomes: A User's Guide. Third edition. Two volumes. (Prepared by the Outcome DEcIDE Center [Outcome Sciences, Inc., a Quintiles company] under Contract No. 290 200500351 TO7.) AHRQ Publication No. 13(14)-EHC111. Rockville, MD: Agency for Healthcare Research and Quality. April 2014. http://www.effectivehealthcare.ahrq.g ov/registries-guide-3.cfm.

6. "Managing Patient Identity across Data Sources." In: Gliklich R, Dreyer N, Leavy M, eds. Registries for Evaluating Patient Outcomes: A User's Guide. Third edition. Two volumes. (Prepared by the Outcome DEcIDE Center [Outcome Sciences, Inc., a Quintiles company] under Contract No. 290 200500351 TO7.) AHRQ Publication No. 13(14)-EHC111. Rockville, MD: Agency for Healthcare Research and Quality. April 2014. http://www.effectivehealthcare.ahrq.g ov/registries-guide-3.cfm.
7. Cusack CM, Knudson AD, Kronstadt JL, Singer RF, Brown AL. Practice-Based Population Health: Information Technology to Support Transformation to Proactive Primary Care (Prepared for the AHRQ National Resource Center for Health Information Technology under Contract No. 290-04-0016.) AHRQ Publication No. 100092-EF. Rockville, MD: Agency for Healthcare Research and Quality. July 2010.

8. Shaljian $M$ and Nielson M. Managing populations, maximizing technology: Population health management in the medical neighborhood. October 2013. Patient-Centered Primary Care Collaborative. https://www.pcpcc.org/downl oad/4378/PCPCC Population Health FINAL e-Version.pdf?redirect=node/200274. Accessed August 2, 2015.

9. Fortini, R, VP and Chief Clinical Officer, Bon Secours Medical System. Personal Communication/Interview on September 12, 2014.

10. Bon Secours Annual Report, 2012. http://hso.bonsecours.com/2012annual-report/files/inc/4a6a937462.pdf. Accessed August 2, 2015.

11. Bon Secours Virginia Medical Group. Patient-Centered Primary Care Collaborative. https://www.pcpcc.org/casestudies/bon-secours. Accessed August 2, 2015.

12. Hines, Scott, MD, Co-Chief Clinical Transformation Officer and Medical Director, Crystal Run Healthcare. Personal Communication/Interview on September 23, 2014.

13. Hong CS, Siegel AL, Ferris TG. Care for high-need, high-cost patients: What makes for a successful care management program. http://www.commonwealthfund.or g/ /media/files/publications/issuebrief/2014/aug/1764_hong_caring_for_high _need_high_cost_patients_ccm_ib.pdf. Accessed August 2, 2015.

14. Arora S, Burner E, Terp S, et al. Improving Attendance at Post-Emergency Department Follow-up Via Automated Text Message Appointment Reminders: A Randomized Controlled Trial. Acad Emerg Med. 2014 
Nov 11. PMID: 25388481. Epub 2014/11/13. Eng.

15. Boland MV, Chang DS, Frazier T, et al. Automated telecommunication-based reminders and adherence with once-daily glaucoma medication dosing: the automated dosing reminder study. JAMA Ophthalmol. 2014 Jul;132(7):845-50. PMID: 24831037. Epub 2014/05/17. eng.

16. Tran N, Coffman JM, Sumino K, et al. Patient reminder systems and asthma medication adherence: a systematic review. J Asthma. 2014 Jun;51(5):536-43. PMID: 24506699. Epub 2014/02/11. eng.

17. Stockwell MS, Westhoff C, Kharbanda EO, et al. Influenza vaccine text message reminders for urban, low-income pregnant women: a randomized controlled trial. Am J Public Health. 2014 Feb;104 Suppl 1:e7-12. PMID: 24354839. Epub 2013/12/21. eng.

18. Matheson EC, Derouin A, Gagliano M, et al. Increasing HPV vaccination series completion rates via text message reminders. J Pediatr Health Care. 2014 JulAug;28(4):e35-9. PMID: 24200295. Epub 2013/11/10. eng.

19. Centers for Medicare \& Medicaid Services. ACO quality measures

[Table]. http://www.cms.gov/Medicare/Med icare-Fee-for-Service-

Payment/sharedsavingsprogram/Downloads/ ACO-Shared-Savings-Program-QualityMeasures.pdf. Accessed August 2, 2015.

20. Peterson M and Muhlestein D. ACO results: What we know so far [Blog post]. May 30, 2014. http://healthaffairs.org/blog/2014/05/30/acoresults-what-we-know-so-far/. Accessed August 2, 2015.

21. James BC, Savitz LA. How Intermountain trimmed health care costs through robust quality improvement efforts. Health Aff (Millwood). 2011 Jun;30(6):1185-91. PMID: 21596758. Epub 2011/05/21. eng.

22. Spalding, J, MD, Medical Director, Enterprise Information Management, Analytics \& Business Intelligence, Divisions of Information Technology \& Medical Operations, Cleveland Clinic. Case report submitted for this article, October 6, 2014.
23. Navaneethan SD, Jolly SE, Schold JD, et al. Development and validation of an electronic health record-based chronic kidney disease registry. Clin J Am Soc Nephrol. 2011 Jan;6(1):40-9.

24. Hasan H. Population health managers, meet the three patient types central to your success [Blog post]. October 23, 2013. http://www.advisory.com/Research/C are-Transformation-Center/CareTransformation-Center-Blog/2013/06/Threecare-delivery-models. Accessed August 2 ,2015.

25. Siegel, M. Managing the risks of healthcare reform: Using risk adjustment and predictive methodologies to drive capitation arrangements, population health management and network performance initiatives. https://www.ncbi.nlm.nih.gov/pu bmed/22787946. Accessed August 24, 2017.

26. Johns Hopkins University. The Johns Hopkins ACG ${ }^{\circledR}$ System. http://acg.jhsph.org/index.php/the-acgsystem-advantage/unique-strengths. Accessed August 2, 2015.

27. Quan H, Li B, Couris CM, et al. Updating and validating the Charlson Comorbidity Index and score for risk adjustment in hospital discharge abstracts using data from 6 countries. Am J Epidemiol. 2011 Mar 15;173(6):676-82.

28. Dubois RW, Feldman M, Lustig A, et al. Are ACOs ready to be accountable for medication use? J Manag Care Pharm. 2014 Jan;20(1):17-21.

29. National Committee for Quality Assurance. Measuring performance in Accountable Care Organizations. 2012. http://www.ncqa.org/Portals/0/Public \%20Policy/2012\%20Updates/NCQA\%20A CO\%20measures\%20Fact\%20Sheet.pdf. Accessed August 2, 2015.

30. Certification Commission for Health Information Technology. A health IT framework for accountable care. http://www.healthit.gov/archive/archive_file s/HIT\%20Policy\%20Committee/2013/Acco untable\%20Care/2013-06-

07/hitpc_aco_wg_june_7_2013_kb.pdf. Accessed August 2, 2015.

31. Hibbard JH, Greene J, Overton V. Patients with lower activation associated with higher 
costs; Delivery systems should know their patients' "scores". Health Aff (Millwood). 2013 Feb;32(2):216-22.

32. Cloudera. Explorys case study with Cloudera \& Hadoop. http://www.cloudera.com/content/cloudera/e n/our-customers/Explorys.html. Accessed August 2, 2015.

33. Brown B and Skelley L. Why population health management strategies need both clinical and claims data. Health Catalyst. http://www.healthcatalyst.com/wpcontent/uploads/2014/07/ClinicalClaims.pdf. Accessed August 2, 2015.

34. Gawande A. The Hot Spotters. The New Yorker Magazine.

2011. http://www.newyorker.com/magazine/ 2011/01/24/the-hot-spotters. Accessed August 2, 2015.

35. Agency for Healthcare Research and Quality. A robust health data infrastructure. (Prepared by JASON The MITRE Corporation under Contract No. JSR-13700.) AHRQ Publication No. 14-0041-EF. Rockville, MD: Agency for Healthcare Research and Quality. April 2014. http://healthit.gov/sites/default/files/pt p13-700hhs white.pdf. 